PACS: $06.60 . \mathrm{Mr}$

\title{
METHOD OF EXPRESS-EVALUATION OF PARAMETERS OF THE SPECTROMETRIC TRACT
}

\author{
S. Reva, O. Turchin, V. Tkach, V. Ananieva \\ V.N. Karazin Kharkiv National University \\ 61022, Kharkiv, 4, Svobody sq., tel/fax 8-057-705-12-47 \\ E-mail: iec-lab@karazin.ua \\ Received 23 October, 2017
}

\begin{abstract}
The method and the scheme of measuring stand are proposed for the fast control of the parameters of spectrometric analog-to-digital converters and other elements of the spectrometric channel. Within a few minutes the stand allows obtaining a qualitative assessment of the main parameters and identifying shortcomings in the operation of the devices that are part of the spectrometer. The method is based on recording and analyzing the spectrum of a sequence of pulses with a uniform amplitude distribution within the input dynamic range of the ADC. The principle of the device operation is described. A brief analysis of the requirements for the parameters of the measuring stand is performed, as well as an analysis of the possibility of determining differential and integral nonlinearity of the ADC, channel profile, noise level estimation in the measuring path with the help of the proposed method. The results of testing of some samples of spectrometric analog-to-digital converters are given and a brief analysis of the results is provided. The possibilities of improving the method are considered for the purpose of obtaining a quantitative estimate of the differential and integral nonlinearity, as well as the noise level of the equipment under test.
\end{abstract}

KEYWORDS: nuclear spectrometry, measurement of ADC parameters, differential nonlinearity of ADC, amplitude spectrum, noise level

\section{МЕТОД ЕКСПРЕС-ОЦІНКИ ПАРАМЕТРІВ СПЕКТРОМЕТРИЧНОГО ТРАКТУ \\ С.М. Рева, О.А. Турчин, В.В. Ткач, В.А. Ананьсва \\ Харківський наиіональний університет імені В.Н. Каразіна \\ 61022, м. Свободи, 4, тел./факс 8-057-705-12-47}

Запропоновано метод та схему вимірювального стенду для швидкого контролю параметрів спектрометричних аналогоцифрових перетворювачів і інших елементів спектрометричного тракту. Стенд дає змогу за кілька хвилин отримати якісну оцінку основних параметрів та виявити недоліки в роботі пристроїв, які входять до складу спектрометру. В основу методу покладено реєстрацію та аналіз спектру послідовності імпульсів 3 рівномірним розподілом амплітуд в межах вхідного динамічного діапазону АЦП. Описано принцип роботи пристрою. Проведено короткий аналіз вимог до параметрів вимірювального стенду, а також аналіз можливості визначення за допомогою запропонованого методу диференційної та інтегральної нелінійності АЦП, профілю каналу, оцінки рівня шумів у вимірювальному тракті. Наведено експериментальні результати тестування деяких зразків спектрометричних аналого-цифрових перетворювачів та проведено короткий аналіз отриманих результатів. Розглянуто можливість удосконалення методу з метою отримання кількісної оцінки диференційної та інтегральної нелінійності, а також рівня власних шумів обладнання, що тестується.

КЛЮЧОВІ СЛОВА: ядерна спектрометрія, вимірювання параметрів АЦП, диференційна нелінійність АЦП, амплітудні спектри, рівень шуму

\section{МЕТОД ЭКСПРЕСС-ОЦЕНКИ ПАРАМЕТРОВ СПЕКТРОМЕТРИЧЕСКОГО ТРАКТА \\ С.Н. Рева, А.А. Турчин, В.В. Ткач, В.А. Ананьева \\ Харьковский национальный университет имени В.Н. Каразина 61022, пл. Свободыл, 4, тел./факс 8-057-705-12-47}

Предложен метод и схема измерительного стенда для быстрого контроля параметров спектрометрических аналогоцифровых преобразователей и других элементов спектрометрического тракта. Стенд позволяет в течение нескольких минут получить качественную оценку основных параметров и выявить недостатки в работе устройств, входящих в состав спектрометра. Метод основан на регистрации и анализе спектра последовательности импульсов с равномерным распределением амплитуд в пределах входного динамического диапазона АЦП. Описан принцип работы устройства. Проведен краткий анализ требований к параметрам измерительного стенда, а также анализ возможностей определения с помощью предложенного метода дифференциальной и интегральной нелинейности АЦП, профиля канала, оценки уровня шумов в измерительном тракте. Приведены результаты тестирования некоторых образцов спектрометрических аналогоцифровых преобразователей и дан краткий анализ полученных результатов. Рассмотрены возможности совершенствования метода с целью получения количественной оценки дифференциальной и интегральной нелинейности, а также уровня собственных шумов тестируемого оборудования.

КЛЮЧЕВЫЕ СЛОВА: ядерная спектрометрия, измерение параметров АЦП, дифференциальная нелинейность АЦП, амплитудные спектры, уровень шумов

\section{АКТУАЛЬНОСТЬ ЗАДАЧИ}

Одной из важнейших задач современной метрологии является регистрация ионизирующих излучений и измерение их энергетического распределения, то есть, регистрация энергетического спектра излучений. Спектральный анализ широко применяется как в экспериментальной ядерной физике, так и для контроля безопасности окружающей среды, в частности - для анализа наличия радиоактивных изотопов в строительных (C) Reva S., Turchin O., Tkach V., Ananieva V., 2017 
материалах, пищевых продуктах и других материалах. Качество и информативность получаемых спектров радиационного излучения в огромной степени зависит от технических параметров отдельных устройств, используемых в составе спектрометрического тракта - детекторов, усилителей сигналов и т.п. Одним из важнейших элементов спектрометра является аналого-цифровой преобразователь. Высокая дифференциальная нелинейность преобразователя может привести к формированию ложных спектральных линий, а наличие шумов приводит к уменьшению разрешающей способности спектрометра.

Занимаясь проектированием спектрометрического оборудования на основе современной элементной базы, коллектив разработчиков лаборатории интеллектуальных электронных систем при кафедре электроники Харьковского национального университета имени В.Н. Каразина столкнулся с задачей оперативного контроля качества параметров создаваемых электронных устройств. Измерение собственного уровня шумов, дифференциальной и интегральной нелинейности аналого-цифрового преобразователя, а также других его параметров выполняется в соответствии с методиками ДСТУ ГОСТ 22252:2008 [1], требует наличия дорогостоящего прецизионного измерительного оборудования и занимает много времени. Но для анализа качества спектрометрического тракта во многих случаях было бы достаточно проведения приближенных (оценочных) измерений, по результатам которых можно было бы сделать выводы о наличии тех или иных технических проблем в тестируемом оборудовании.

Целью данной работы является изучение возможности оценки основных технических параметров спектрометрического тракта на основе анализа спектра, получаемого в процессе формирования и регистрации тестового сигнала с равномерным амплитудным распределением импульсов.

\section{ТЕХНИЧЕСКАЯ РЕАЛИЗАЦИЯ ИЗМЕРИТЕЛЬНОЙ СХЕМЫ}

Авторами данной статьи для решения поставленной задачи была предложена методика с использованием простой измерительной установки, схема которой показана на рис. 1.

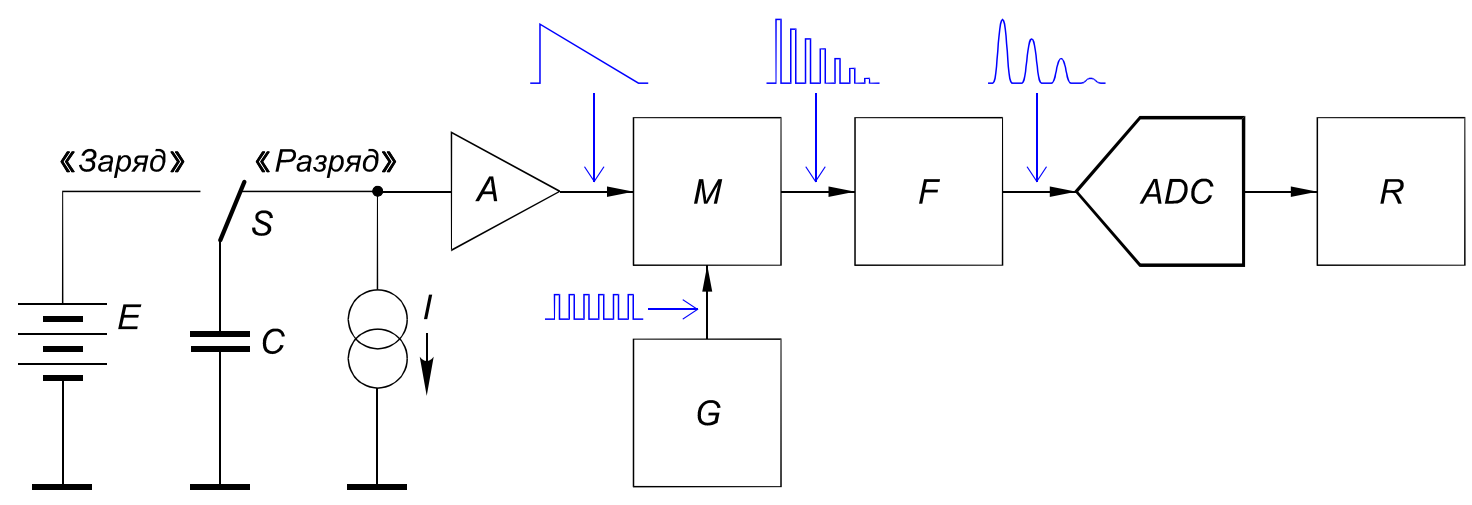

Рис. 1. Функциональная схема измерительной установки

Е - источник питания; S - переключатель; I - источник стабильного тока; C - конденсатор; A - повторитель напряжения; $\mathrm{G}$ - генератор импульсов; M - импульсный модулятор; F - формирователь импульсов; ADC - тестируемый аналогоцифровой преобразователь; $\mathrm{R}$ - регистратор спектра

Установка состоит из конденсатора С емкостью в несколько сотен микрофарад, который перед началом эксперимента заряжается от источника питания до некоторого начального значения напряжения. После перевода переключателя S в положение «Разряд» начинается разряд конденсатора стабилизированным током, в результате чего напряжение на нем медленно снижается по линейному закону. При токе разряда $30 \ldots 50$ мкА весь процесс разряда проходит в течении нескольких минут. Высокая линейность формируемого аналогового сигнала при условии малых собственных токов утечки конденсатора может быть обеспечена за счет высокой стабильности генератора тока I и повторителя напряжения А, построенных на основе современных операционных усилителей с соответствующими параметрами. Линейно изменяющееся напряжение поступает на вход импульсного модулятора М, который выполнен на основе аналогового коммутатора на МОП-транзисторах. Частота модуляции и длительность импульсов задается с помощью генератора с кварцевой стабилизацией частоты. Полученная последовательность импульсов с плавно изменяющейся амплитудой проходит через дифференцирующе-интегрирующую формирующую цепь $\mathrm{F}$, собранную на пассивных элементах. В результате импульсы приобретают форму, напоминающую форму функции Гаусса, после чего могут быть поданы на вход тестируемого спектрометрического АЦП. Отсутствие в цепи формирования импульсного сигнала каких-либо усилительных элементов и p-n-переходов обеспечивает высокую стабильность коэффициента передачи схемы, а использование элементов с низким коэффициентом температурной зависимости дает возможность пренебречь незначительными колебаниями температуры окружающей среды во время проведения измерений. Для регистрации спектра применяется персональный 
компьютер или контроллер спектрометра со штатным программным обеспечением (на рисунке обозначен как регистрирующее устройство R).

В первом варианте конструкции измерительной установки для проверки самой идеи метода в качестве накопительных конденсаторов были применены танталовые объемно-пористые электролитические конденсаторы. Они имеют токи утечки, величина которых зависит от напряжения на конденсаторах. Это приводит к нелинейности процесса разряда. Скорость разряда увеличивается при напряжении более $10 . .12$ В и уменьшается при напряжении менее 3 В. Наблюдается также эффект тренировки конденсаторов, который приводит к уменьшению нелинейности разряда в области высоких напряжений после нескольких циклов повторного заряда. Тем не менее, при всех указанных недостатках с помощью этой установки удалось провести целый ряд сеансов тестирования, которые предоставили разработчикам полезную информацию о параметрах некоторых спектрометрических аналого-цифровых преобразователей.

На рис. 2 показан амплитудный спектр, который получен в результате тестирования аналого-цифрового преобразователя «АЦП Спк» (г. Дубна, 2007 г.). Преобразователь построен на основе интегрального АЦП $\mathrm{AD} 7892$, который является двенадцатиразрядным аналого-цифровым преобразователем последовательного приближения. Регистрация спектра выполнялась с помощью промышленного компьютера Advantech PCM-3343 и спектрометрической программы «Omega».

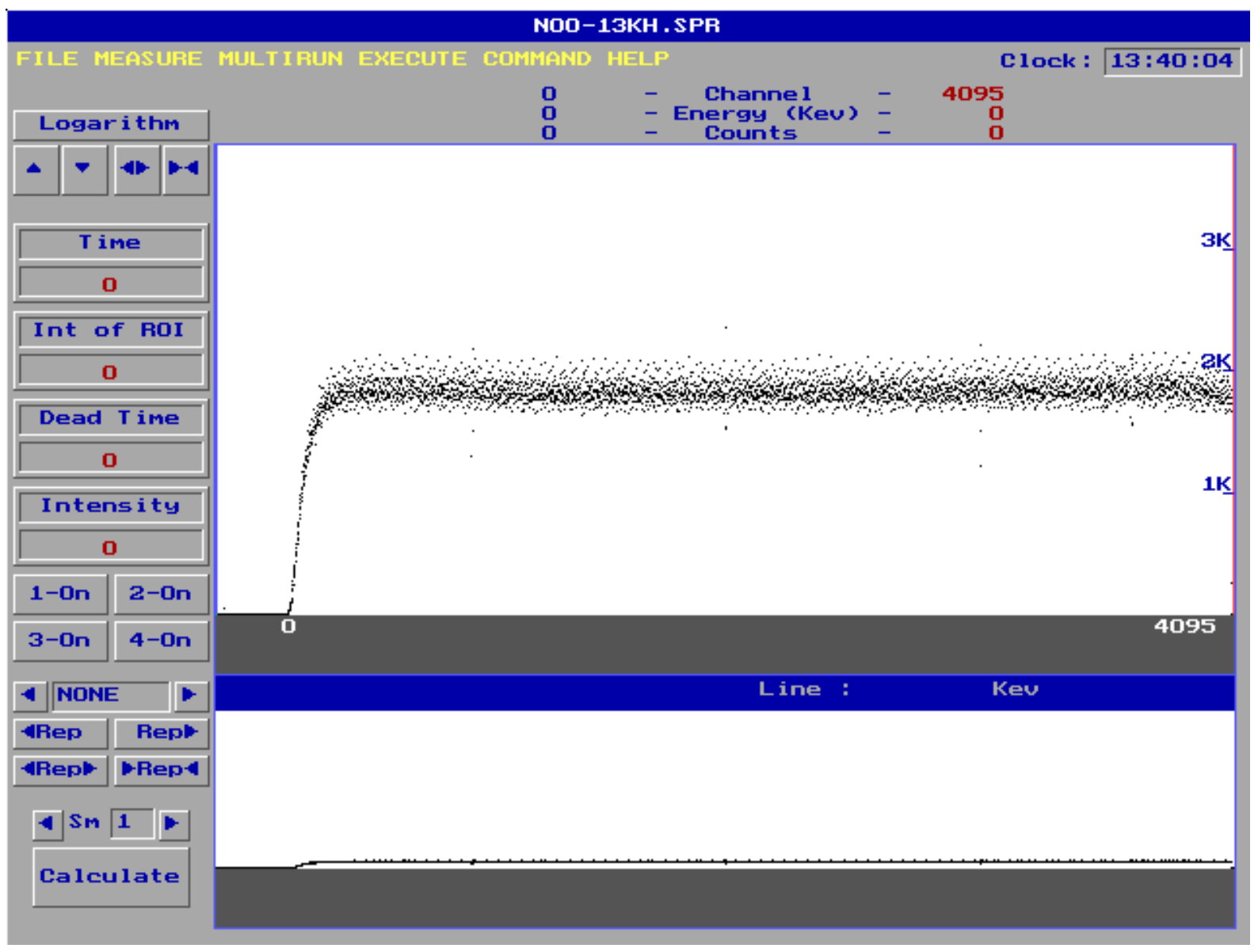

Рис. 2. Амплитудный спектр, полученный при тестировании АЦП последовательного приближения

Измерения проводились при начальном напряжении заряда конденсаторов около 18 В, емкости конденсаторов 750 мкФ и частоте следования импульсов 13 кГц. Амплитуда импульсов на выходе формирователя при этом изменялась от 4 В до 0 В. При выбранной частоте модулирующих импульсов и токе разряда конденсатора в каждом канале спектра регистрировалось в среднем около 1800 импульсов.

Как видно из рисунка, на спектре присутствуют явно выраженные периодические отклонения отдельных точек от усредненной линии спектра, которые свидетельствуют о наличии дифференциальной нелинейности АЦП. Наиболее заметны отклонения при переходе из одной четверти диапазона напряжений в другую.

На рис. 3 подробно показан небольшой участок амплитудного спектра этого же АЦП. Левый маркер установлен на позиции канала 254. Нетрудно заметить, что количество импульсов, зарегистрированных в следующих двух каналах, существенно отличается от среднего значения. В канале 255 импульсов зарегистрировано заметно меньше среднего значения, а в канале 256 - заметно больше (на рисунке эти точки выделены прямоугольниками). 


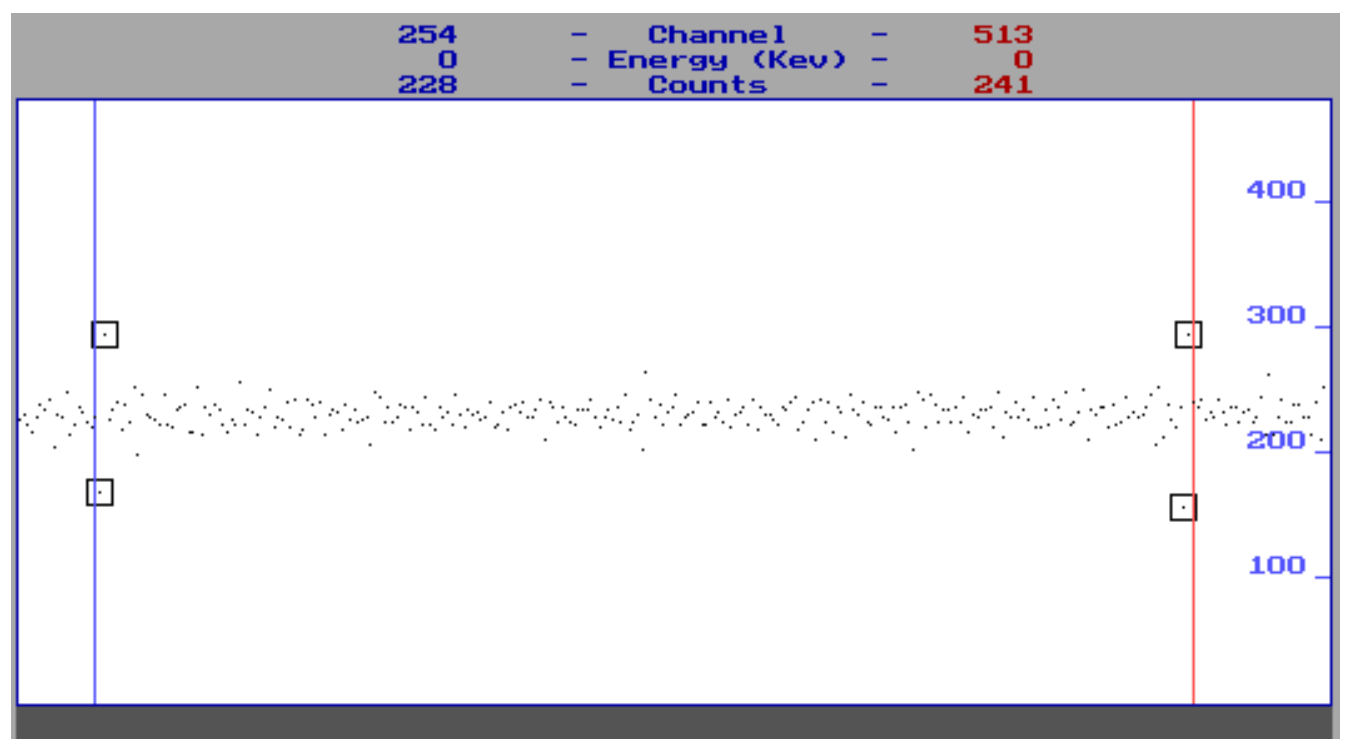

Рис. 3. Фрагмент амплитудного спектра АЦП последовательного приближения

Аналогичная ситуация наблюдается в каналах 511 и 512 (правый маркер установлен на канал 513). Координаты этих точек в спектре соответствуют переключению старших разрядов АЦП последовательного приближения, что обусловлено неточностью изготовления элементов резисторной матрицы в составе интегрального АЦП. Оценка разброса ширины каналов по абсолютным значениям сформированного спектра показывает, что дифференциальная нелинейность данного преобразователя в отдельных точках превышает $25 \%$, то есть, ширина некоторых каналов более чем на $25 \%$ отличается от средней ширины канала АЦП. Об этом свидетельствует заметно увеличенное или уменьшенное количество импульсов, зарегистрированных в этих каналах при равномерной плотности распределения их амплитуд. Спектр также характеризуется достаточно большим случайным непериодическим разбросом точек. Это может быть результатом воздействия на входной сигнал собственных шумов АЦП, в том числе и цифровых шумов интерфейсной части преобразователя.

\section{АНАЛИЗ ПРОЦЕССА ФОРМИРОВАНИЯ СПЕКТРА}

Чтобы понять, каким образом те или иные параметры АЦП и других элементов спектрометрического тракта влияют на форму результирующего амплитудного спектра, проведем анализ процесса его формирования.

Измерительная установка, схема которой показана на рис. 1, в идеальном случае генерирует последовательность импульсов $i$ с равномерно уменьшающейся амплитудой, $m$ импульсов из которых попадает в динамический диапазон АЦП (от 0 до $U_{\max }$, рис. 4).

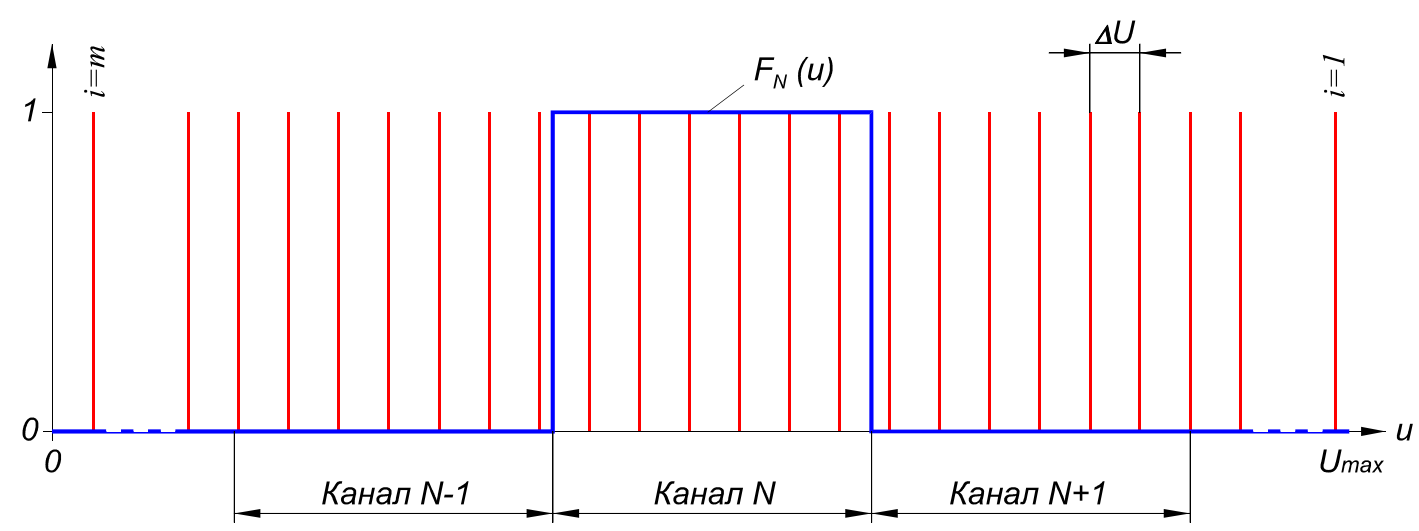

Рис. 4. Диаграмма распределения вероятностей

Если скорость разряда конденсатора составляет $s$ (мВ/с), то при коэффициенте передачи формирователя импульсов $\mathrm{F}$ (рис. 1) равным $K_{F}$ и при частоте импульсной модуляции $F_{M}$ (кГц) изменение амплитуды каждого импульса по сравнению с предыдущим в микровольтах составит:

$$
\Delta U=s \cdot K_{F} / F_{M} .
$$


При высокой стабильности частоты модулирующего генератора и постоянной скорости изменения входного напряжения функции вероятности генерации очередных импульса будут приближены к $\delta$-функциям (на рис. 4 показаны в виде вертикальных отрезков), математическое ожидание которых $U i$ может быть найдено как

$$
U_{i}=U_{\max }-\Delta U(i-1) \cdot 10^{-6},
$$

где $i$ - номер импульса в последовательности, $i=1$ соответствует первому импульсу с наибольшей амплитудой $U_{\max }(\mathrm{B})$, попавшему в диапазон входных напряжений АЦП.

Предположим, что АЦП имеет идеальные метрологические характеристики, а именно - нулевую интегральную и дифференциальную нелинейность. В этом случае весь входной динамический диапазон напряжений АЦП разделяется на некоторое количество каналов (поддиапазонов) одинаковой ширины, общее число которых определяется разрядностью преобразователя. Как правило, общее количество каналов $N_{\max }=2^{k}$, где $k$ - разрядность АЦП. Ширина канала в этом случае в микровольтах составит:

$$
H=10^{6} \cdot U_{\max } / N_{\max } .
$$

Функция вероятности попадания импульса с амплитудой $U_{i}$ в канал $N$ показана на рисунке 4 в виде сплошной жирной линии. В пределах канала вероятность равна единице, а за его пределами - нулю.

На первый взгляд может показаться, что при постоянной частоте модуляции и постоянной скорости изменения напряжения на конденсаторе в каждый измерительный канал будет попадать одинаковое количество импульсов, а результирующий амплитудный спектр будет представлять собой множество точек, расположенных на одной горизонтальной линии. Такой вариант формирования спектра теоретически возможен только при четкой синхронизации процесса разряда конденсатора с частотой импульсного генератора. При этом общее количество импульсов, попадающих в динамический диапазон АЦП, должно без остатка делиться на количество каналов. По причине отсутствия в предлагаемой измерительной схеме механизмов синхронизации упомянутых процессов, а также из-за наличия интегральной нелинейности как тестируемого АЦП, так и самого измерительного устройства, получить идеальный спектр на практике невозможно. Из-за постоянно изменяющегося фазового набега импульсов по отношению к моментам времени, когда линейно изменяющаяся во времени функция амплитуды пересекает границу соседних каналов, количество импульсов, попадающих в один канал может изменяться на единицу даже в случае тестирования идеального АЦП. Это наглядно иллюстрирует рисунок 4: при одинаковой ширине измерительных каналов и абсолютно равномерном распределении импульсов по амплитуде в канал $\mathrm{N}$ попадает шесть импульсов, а в канал $\mathrm{N}-1$ - семь импульсов.

Следовательно, спектр идеального АЦП при предлагаемом методе тестирования будет представлять собой множество точек, которые отстоят от некоторой горизонтальной линии не более чем на единицу.

\section{Оценка дифференциальной нелинейности}

Как видно из результатов тестирования АЦП последовательного приближения (рис. 2), предлагаемый метод может быть использован для выявления дифференциальной нелинейности спектрометрического преобразователя.

В диапазоне измерения от 0 В до некоторого значения $U_{\max }$ измерительным устройством будет выработано $m$ импульсов, при этом

$$
m=U_{\max } \cdot 10^{6} / \Delta U \text {. }
$$

Подставив (1) в (4), получим:

$$
m=U_{\max } \cdot 10^{6} \cdot F_{M} /\left(s \cdot K_{F}\right) .
$$

В этом случае количество импульсов $X$, попадающих в один канал составит:

$$
X=m / N_{\max },
$$

или в некоторых каналах:

$$
X=\left(m / N_{\max }\right)+1
$$

при условии, что $m$ не делится без остатка на $N_{\max }$.

То есть, для идеального АЦП, имеющего нулевую дифференциальную нелинейность, количество импульсов, зарегистрированных в каждом из каналов, будет соответствовать значению

$$
X=0,5+\left(m / N_{\max }\right)+\varepsilon,
$$


где $\varepsilon$ - неопределенность, с некоторой степенью вероятности численно равная значению $+0,5$ или $-0,5$.

Из-за наличия неопределенности $\varepsilon$ практически невозможно будет по внешнему виду амплитудного спектра распознать дифференциальную нелинейность АЦП, если она приводит к расширению или сужению отдельных каналов на величину менее чем $\Delta U$. При отличии ширины $i$-го канала от среднего значения ширины канала $\bar{H}$ на величину $\Delta U$ дифференциальная нелинейность [2] этого канала составит:

$$
K_{D}(i)=\frac{H_{i}-\bar{H}}{\bar{H}} \cdot 100 \%=\frac{\Delta U}{\bar{H}} \cdot 100 \% .
$$

Следовательно, исходя из выражения (6), для определения факта наличия некоторой дифференциальной нелинейности величиной $K_{D M}$ необходимо обеспечить условие:

$$
\Delta U<\bar{H} \cdot \frac{K_{D M}}{100 \%},
$$

где $K_{D M}$ - порог обнаружения дифференциальной нелинейности. С учетом формул (1) и (7) получим:

$$
\frac{s \cdot K_{F}}{F_{M}}<\bar{H} \cdot \frac{K_{D M}}{100 \%}
$$

Из выражения (8) находим:

$$
\frac{1}{F_{M}}<\frac{\bar{H}}{s \cdot K_{F}} \cdot \frac{K_{D M}}{100 \%} .
$$

Левая часть неравенства (9) представляет собой период следования модулирующих импульсов в миллисекундах, так как частота $F_{M}$ в формуле (1) задана в килогерцах, обозначим его как $\tau$. Если среднее значение ширины канала $\bar{H}$ принять в соответствии с формулой (3), то выражение (9), которое является необходимым условием для обнаружения дифференциальной нелинейности величиной $K_{D M}$, приобретает вид:

$$
\tau<\frac{U_{\max }}{s \cdot K_{F} \cdot N_{\max }} \cdot \frac{K_{D M}}{100 \%} \cdot 10^{6} .
$$

Предположим, что при тестировании АЦП накопительный конденсатор С (рис. 1) емкостью 500 мкФ, разряжается током $I_{D C}=50$ мкА. В этом случае скорость разряда конденсатора составит:

$$
s=I_{D C} / C=50 \cdot 10^{-6} / 500 \cdot 10^{-6}=0,1(\mathrm{~B} / \mathrm{c})=100(\mathrm{mB} / \mathrm{c}) .
$$

Если конденсатор был предварительно заряжен до напряжения $U_{0}=15 \mathrm{~B}$, то общее время разряда конденсатора составит 150 секунд. При коэффициенте передачи амплитуды формирователя импульсов $K_{F}=1 / 3$ амплитуда выходных импульсов будет изменяться в пределах входного диапазона напряжений АЦП от $U_{\max }=5 \mathrm{~B}$ до $0 \mathrm{~B}$. В этом случае, например, для обнаружения дифференциальной нелинейности на уровне $K_{D M}=1 \%$ при тестировании спектрометрического двенадцатиразрядного АЦП ( $N_{\max }=4096$ каналов) в соответствии с выражением (10) период следования модулирующих импульсов не должен превышать 0,366 мс, то есть, частота модулирующего генератора должна составлять не менее 2,73 кГц.

Следует отметить, что условие (10) справедливо только для случая отсутствия как собственных шумов АЦП, так и случайных (шумовых) составляющих в составе спектра тестирующего сигнала.

\section{Влияние шумов на амплитудный спектр}

Если на тестовый сигнал, представляющий собой периодическую последовательность импульсов, амплитуда которых уменьшается с постоянной скоростью, накладывается сигнал шума, то амплитуда каждого очередного импульса не будет в точности соответствовать ее математическому ожиданию (2), а функция вероятности появления сигнала с заданной амплитудой не будет представлять собой $\delta$-функцию. В результате воздействия сигнала шума с нормальным законом распределения вероятности функция вероятности амплитуды импульса также приобретает форму кривой Гаусса (рис. 5). Математическое ожидание этой функции по прежнему может быть найдено по формуле (2), а дисперсия будет зависеть от суммарного уровня шума, пересчитанного ко входу аналого-цифрового преобразователя. 


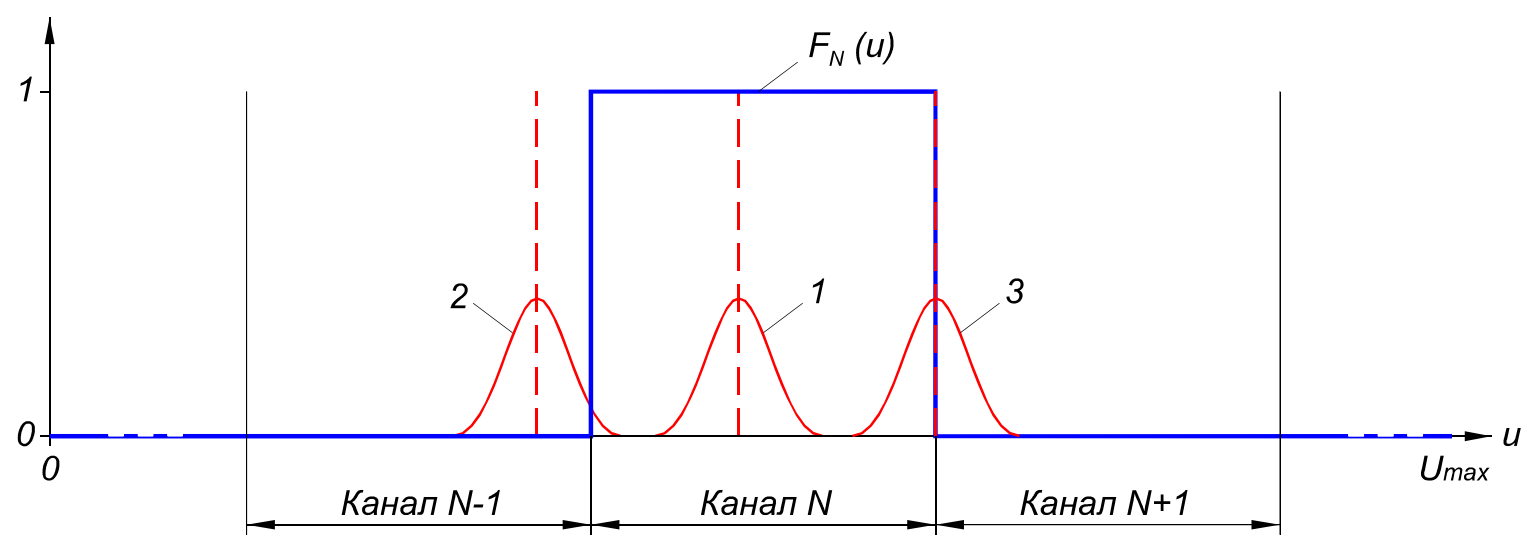

Рис. 5. Влияние шума на вероятность попадания в канал

Если действующее значение шума заметно меньше ширины канала, то неопределенность, обусловленная воздействием шумового сигнала, не будет приводить к перераспределению вероятности попадания в заданный канал при условии, что математическое ожидание функции распределения вероятности попадает в зону, близкую к середине канала (кривая 1 на рис. 5). Если же воздействию такого же самого уровня шума будет подвергнут тестовый сигнал, амплитуда которого близка к границе канала, появляется вероятность регистрации этого сигнала в соседнем канале (кривая 2). При условии совпадения математического ожидания с границей двух соседних каналов регистрация события в каждом из них становится равновероятной (кривая 3).

Воздействие на входной сигнал шума, возникающего во входных цепях АЦП, приводит к искажению формы функций вероятности попадания в каналы. Они приобретают вид, показанный на рис. 6 . Для описания формы функции вероятности в спектрометрии используется характеристика, которая носит название профиля канала [3]. Понятно, что профиль канала и его эффективная ширина непосредственно зависят от уровня собственных шумов аналого-цифрового преобразователя. Поэтому, определив один из этих параметров АЦП, можно сделать вывод о величине второго связанного с ним параметра.

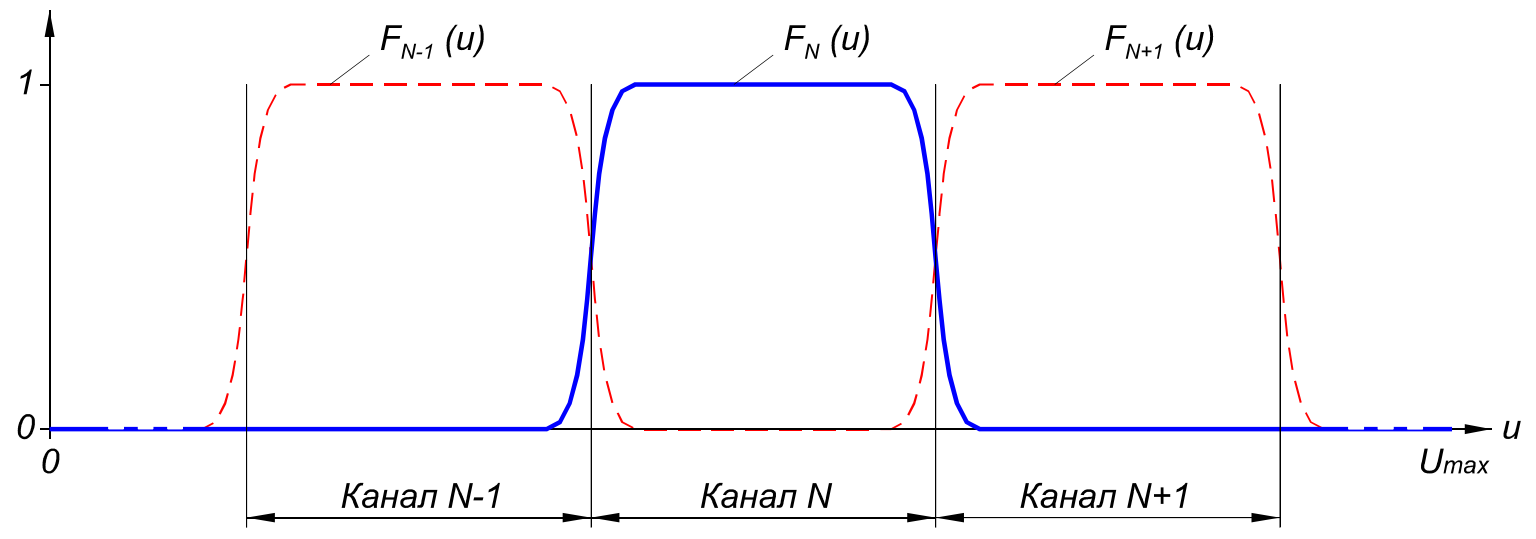

Рис. 6. Формирование профиля канала при наличии шумов

В результате случайного воздействия шумового сигнала на последовательность тестовых импульсов возникает частичное перераспределение попаданий амплитуд в отдельные каналы аналого-цифрового преобразователя. Линия амплитудного спектра становится «размытой» случайными отклонениями количества зарегистрированных в том или ином канале импульсов от их среднего значения. Закономерно предположить существование зависимости степени разброса значений амплитудного спектра от уровня собственных шумов измерительного тракта. Эта статистическая зависимость может быть найдена аналитически, экспериментально или методом численного моделирования измерительной системы.

\section{Искажения амплитудного спектра за счет интегральной нелинейности}

Интегральная нелинейность АЦП возникает как результат интегрального накопления на определенных участках передаточной характеристики дифференциальной нелинейности отдельных каналов, если они имеют один и тот же знак. Другими словами, изгиб передаточной характеристики АЦП возникает из-за того, что на ее некоторых участках каналы имеют ширину, которая чуть больше среднего значения ширины канала, а на других участках - чуть меньше среднего. Понятно, что при тестировании такого АЦП с помощью предложенной методики средняя линия амплитудного спектра будет иметь более высокий уровень в области расположения более широких каналов и более низкий - в области зауженных каналов. То есть, статистические 
характеристики амплитудного спектра, полученного в результате регистрации тестового сигнала, содержат в себе информацию и об интегральной нелинейности АЦП. Для ее оценки необходимо в первую очередь обеспечить высокую линейность процесса разряда накопительного конденсатора. Применение электролитических конденсаторов в этом случае становится недопустимым из-за явно выраженной зависимости токов утечки от значения приложенного напряжения.

Для количественной оценки интегральной нелинейности весь диапазон амплитуд тестового сигнала должен быть разбит на несколько участков с последующим определением среднего значения уровня спектральной линии на каждом из участков. Эта задача может быть решена программными средствами путем статистического анализа спектрометрического массива данных.

\section{ПРИМЕРЫ ПРИМЕНЕНИЯ МЕТОДА ЭКСПРЕСС-ОЦЕНКИ}

Разработанная авторами статьи измерительная установка использовалась для проверки работы некоторых образцов аналого-цифровых преобразователей, а также применялась для экспресс-контроля параметров создаваемых в лаборатории спектрометрических АЦП.

На рис. 7 приведен фрагмент амплитудного спектра, полученного с помощью измерительного стенда на одном из экспериментальных образцов АЦП последовательного счета, цифровой узел которой построен на основе программируемой логической матрицы (ПЛМ) серии МАХ 7000.

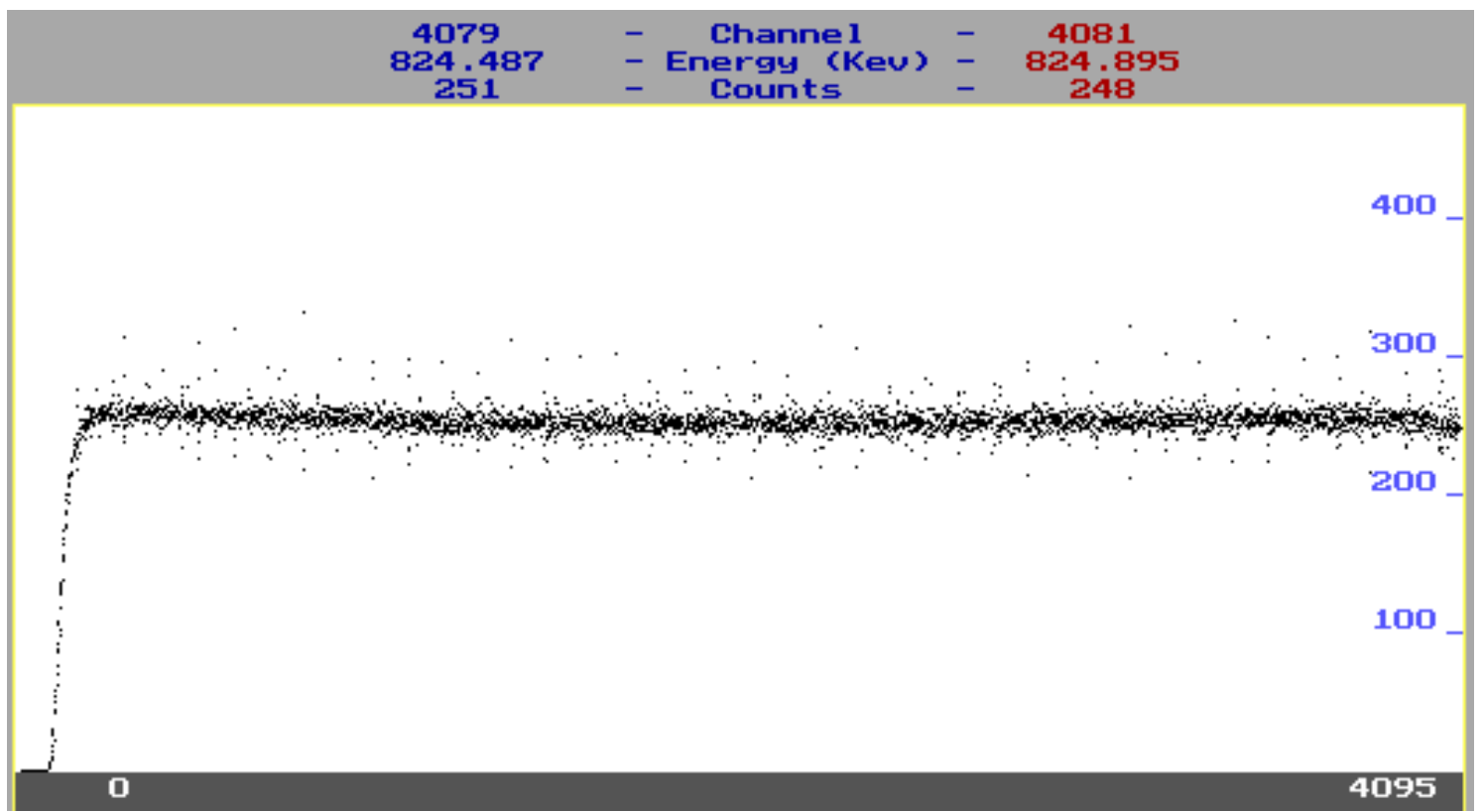

Рис. 7. Амплитудный спектр АЦП на основе ПЛМ серии МАХ7000

Как видно из рисунка, на спектре присутствуют периодически повторяющиеся отклонения отдельных точек, свидетельствующие о наличии в некоторых каналах достаточно большой дифференциальной нелинейности, что не характерно для аналого-цифровых преобразователей последовательного счета. Обнаруженный с помощью предлагаемого метода экспресс-оценки недостаток послужил поводом для проведения ряда исследований, в ходе которых было выявлено, что причиной возникновения периодически повторяющейся дифференциальной нелинейности является технологическая особенность программируемых логических матриц этой серии. Характерные для них большие коммутационные токи при переключении триггеров приводят к возникновению взаимного влияния между макроячейками ПЛМ. Текущее состояние матрицы влияет на порог переключения входных логических элементов, что приводит к паразитной фазовой модуляции тактовой частоты, поступающей на счетчик. Этот процесс является детерминированным и полностью зависит от состояния отдельных триггеров счетчика. В результате возникает обнаруженная при тестировании дифференциальная нелинейность, которая с высокой стабильностью регистрируется от измерения к измерению в одних и тех же каналах. Для устранения обнаруженного недостатка разработчиками были внесены некоторые изменения в принципиальную схему АЦП, а также использована другая серия программируемых логических матриц.

Вторым примером применения разработанного метода оценки является тестирование спектрометрического преобразователя, который построен на основе микроконтроллера С8051F060. Измерения в нем выполняются с помощью встроенного в микроконтроллер АЦП последовательного приближения, для уменьшения дифференциальной нелинейности АЦП использован метод «скользящей шкалы» [4].

На рис. 8 показан фрагмент амплитудного спектра, полученного при использовании метода экспрессоценки с помощью штатной спектрометрической программы, которой разработчик комплектует данный АЦП. 


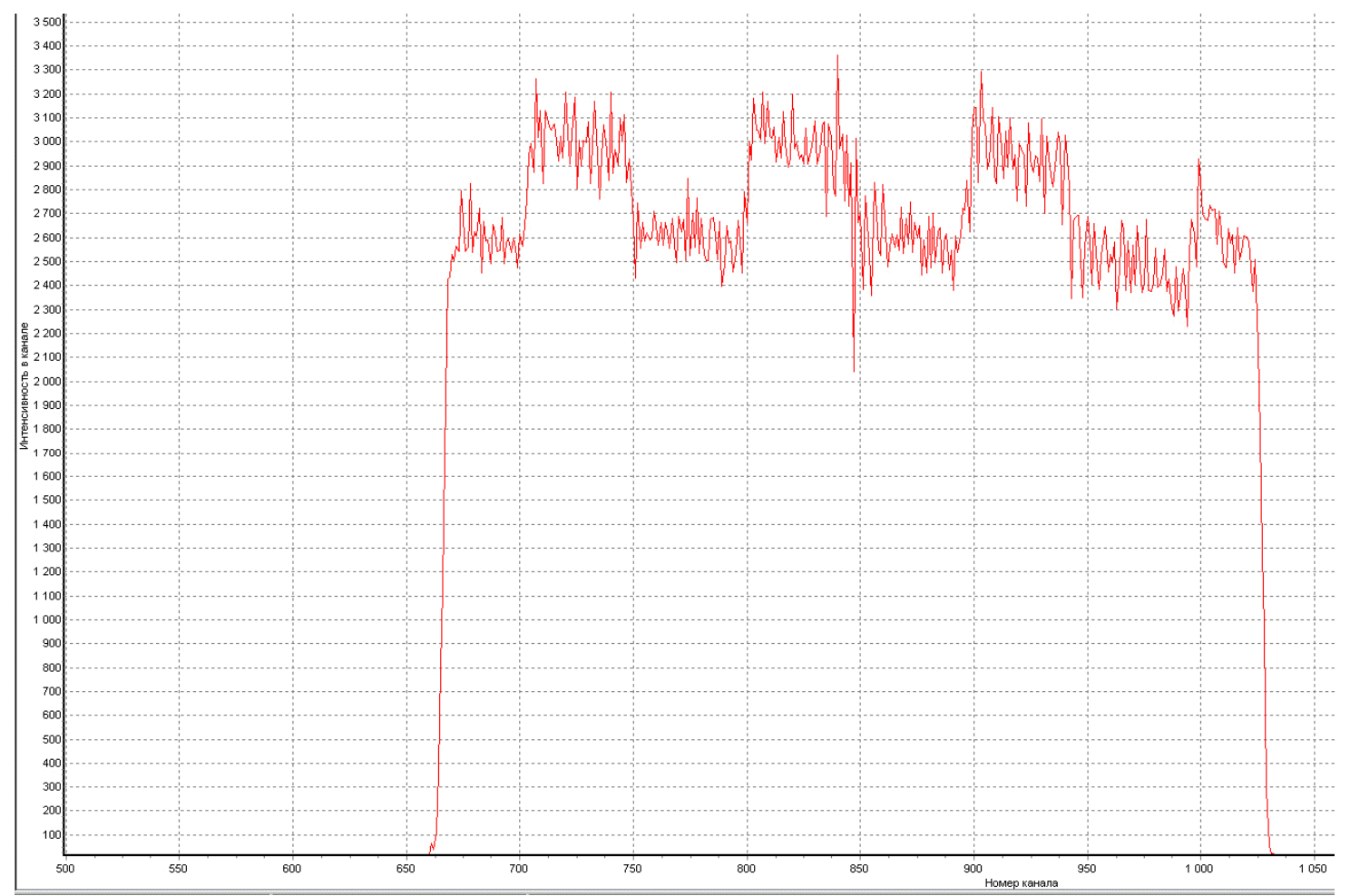

Рис. 8. Амплитудный спектр, полученный при тестировании АЦП на основе микроконтроллера C8051F060

Анализируя форму полученного спектра, можно сделать следующие выводы:

1) в тестируемом АЦП реализация метода «скользящей шкалы» выполнена с явными аппаратными или алгоритмическими ошибками, что приводит к возникновению периодического излома формируемой спектральной линии (в виде меандра);

2) конструктивное решение, основанное на применении встроенного в микроконтроллер аналогоцифрового преобразователя, является неприемлемым для спектрометрии, так как высокий уровень цифровых шумов микроконтроллера существенно воздействует на полезный сигнал и, как результат, приводит к возникновению заметного стохастического разброса точек, образующих линию спектра.

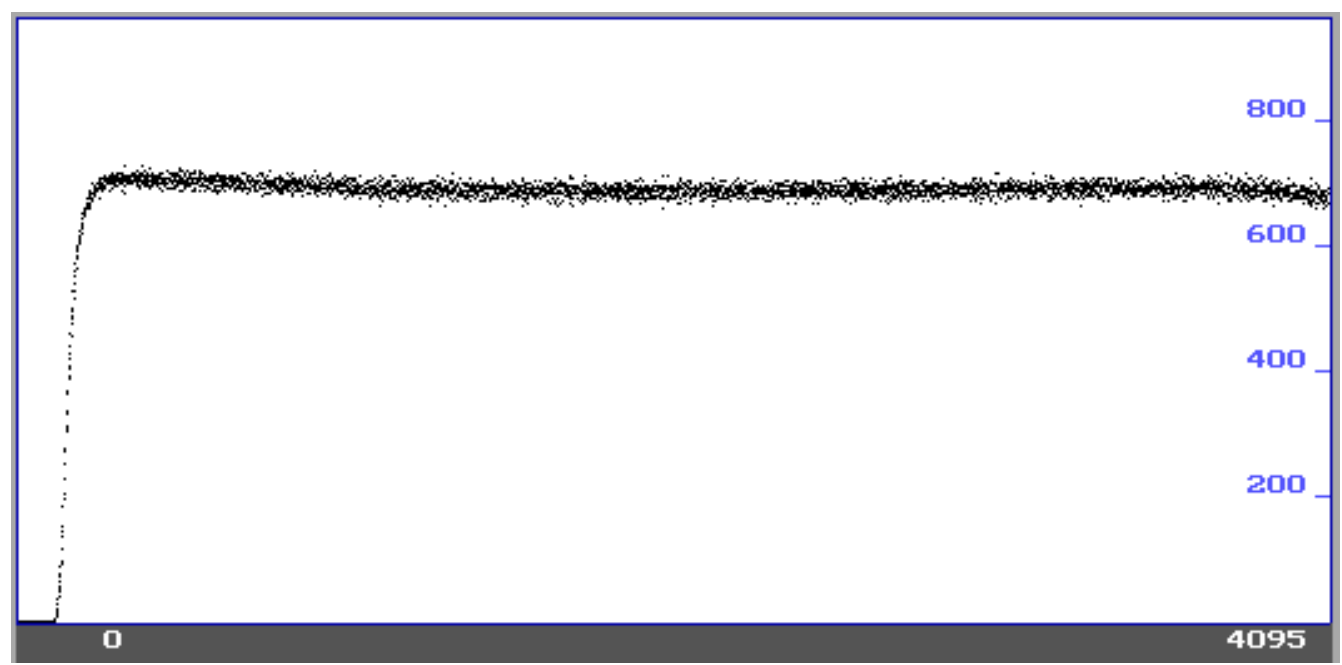

Рис. 9. Амплитудный спектр, полученный при тестировании АЦП Вилкинсона при частоте модуляции 5 кГц

Одной из возможностей тестирования предложенным экспресс-методом является оценка уровня собственных шумов аналого-цифрового преобразователя. На рис. 9,10 показаны амплитудные спектры, полученные на одном и том же аналого-цифровом преобразователе последовательного счета (АЦП Вилкинсона), но при разных значениях частоты модулирующих импульсов.

Спектр на рис. 9 соответствует частоте модуляции 5 кГц. При данной частоте в каждый из каналов попадает около 700 тестовых импульсов с плавно изменяющейся амплитудой. Спектр представляет собой 
горизонтальную линию (если не брать во внимание наклона на начальном и конечном участках спектра из-за наличия переменных токов утечки электролитических конденсаторов), которая «размыта» отклонениями значений, полученных в отдельных каналах, от некоторого среднего значения. Разброс точек вызван наличием шума во входных цепях АЦП, причиной которого может быть в том числе шум самих электролитических конденсаторов.

О том, что данный разброс значений не является следствием дифференциальной нелинейности свидетельствует результат следующего эксперимента, который был выполнен при частоте модулирующих импульсов около 19 кГц (рис. 10).

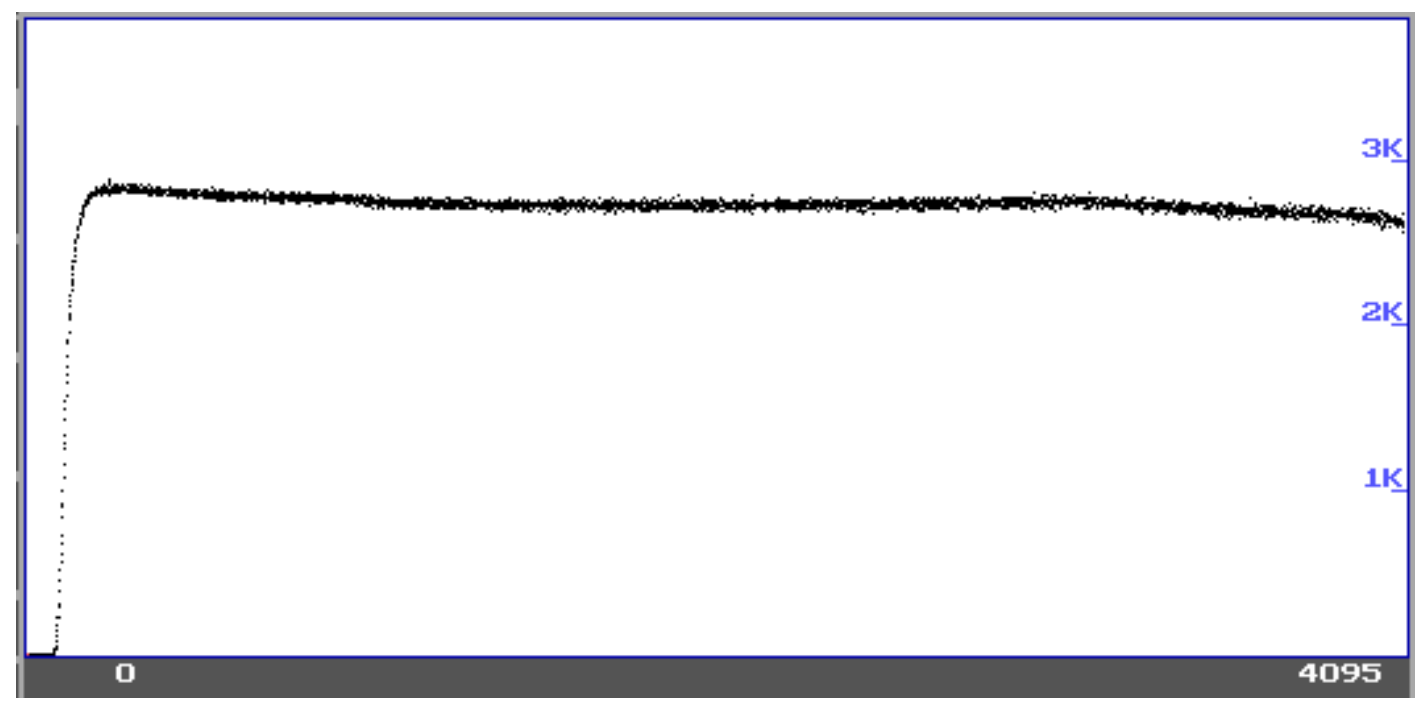

Рис. 10. Амплитудный спектр, полученный при тестировании АЦП Вилкинсона при частоте модуляции 19 кГц

Среднее значение импульсов, зарегистрированных в каждом из каналов, возросло примерно в четыре раза и составляет около 2800 импульсов на канал. Нетрудно заметить, что на этом амплитудном спектре относительный разброс точек от среднего значения уменьшился примерно в два раза, что соответствует квадратному корню от коэффициента увеличения количества импульсов на канал по сравнению с предыдущим экспериментом. Это свидетельствует о стохастическом характере процессов, вызывающих размывание спектральной линии. При наличии явно выраженной дифференциальной нелинейности относительное отклонение значений, получаемых в отдельных каналах, не должно зависеть от частоты модуляции, используемой при проведении эксперимента.

\section{ЗАКЛЮЧЕНИЕ}

Эксперименты с разными аналого-цифровыми преобразователями, проведенные на разработанном авторами измерительном стенде, показали возможность применения данного метода экспресс-тестирования для оценки основных технических параметров как самих аналого-цифровых преобразователей, так и некоторых других элементов спектрометрического тракта. Метод удобен для контроля параметров оборудования как на существующих измерительных комплексах, так и в процессе разработки новых устройств для спектрометрических трактов, в первую очередь - спектрометрических АЦП и каскадов предварительного усиления и формирования сигналов, поступающих от детекторов. На основе полученных экспериментальных результатов можно сделать выводы о возможных направлениях дальнейшего совершенствования методики измерения и самого измерительного стенда.

Во-первых, явным недостатком стенда является применение электролитических конденсаторов в качестве накопителя заряда, так как собственные токи утечки приводят к нарушению линейности процесса разряда и искривлению спектральной линии, что наблюдается при тестировании практически всех моделей спектрометрических АЦП. Для устранения этого недостатка необходимо заменить в составе стенда используемые электролитические конденсаторы конденсаторами с малыми токами утечки, например, пленочными на основе диэлектрика из полипропилена или полиэтилентерефталата.

Во-вторых, следует отметить, что информационность получаемых на стенде амплитудных спектров позволяет не только выявлять недостатки измерительных устройств, но и получать приближенные численные значения некоторых основных параметров аналого-цифровых преобразователей.

О величине интегральной и дифференциальной нелинейности и уровне шума свидетельствуют отклонения отдельных точек или участков спектральной линии от некоторого среднего значения. Для получения численной информации о каждом из этих параметров необходимо провести ряд дополнительных теоретических и практических исследований с целью определения корреляционной зависимости между отдельными 
параметрами и характеристиками спектрометрического АЦП и статистическими характеристиками получаемых спектров. Для этого необходимо:

1) ввести критерии для оценки разброса точек на отдельных участках формируемой в процессе измерения спектральной линии;

2) построить аналитическую или численную модель процесса регистрации спектра на предложенном измерительном стенде;

3) на основе полученной модели процесса определить корреляционные зависимости между статистическими параметрами регистрируемых спектров и отдельными характеристиками и параметрами спектрометрического АЦП;

4) разработать программное обеспечение, позволяющее автоматизировать процесс оценки параметров АЦП по результатам экспресс-тестирования на измерительном стенде;

5) выполнить экспериментальную проверку модифицированного измерительного стенда совместно с программным обеспечением и выработать рекомендации по использованию предложенного метода контроля параметров спектрометрических АЦП.

\section{СПИСОК ЛИТЕРАТУРЫ}

1. DSTU GOST 22252:2008: Multichannel amplitude analyzers. Methods of parameter measurement - [Effective from 2008-1001]. - Kyiv: Derzhspozhyvstandart of Ukraine, 2008. - 14 p. - (National standard of Ukraine). (in Ukraine)

2. Gavrilov L.E. Fundamentals of Nuclear Electronics. Part 1. - Moscow: National Research Nuclear University "MEPhI", 2010. P. 150,154. (in Russian)

3. Sheremetyev A.K. Recommendations for the use of the spectrometric ADC [Electronic resource] // Scintillators.ru. https://scintillators.ru/booc/scintillators.ru-adc.pdf. (in Russian)

4. Aulchenko V.M., Ponomarev S.A. Bitwise-balancing analog-to-digital converter with a small differential nonlinearity. Novosibirsk: Budker Institute of Nuclear Physics Siberian Branch of USSR Academy of Science, 1982. - 7p. - (Preprint Budker Institute of Nuclear Physics Siberian Branch of USSR Academy of Science; BINP SB USSR of AS 1982). (in Russian) 